\title{
Occupational Health Hazards among Health Care Workers
}

\author{
Nazanin Izadi* and Reza Piruznia \\ Tehran University of Medical Sciences, Iran
}

*Corresponding author: Nazanin Izadi, Center for Research on Occupational Diseases, Tehran University of Medical Sciences, Tehran, Iran, Tel: +98 21 6640558; Email:

\section{Editorial}

Volume 2 Issue 1

Received Date: April 05, 2018

Published Date: April 16, 2018

DOI: $10.23880 /$ phoa- 16000120

nazanin.izadi@gmail.com

Health care industry around the world employ over 43 million health workers in 2015, including 9.8 million physicians and 20.7 million nurses/midwives [1]. Hospital staffs are exposed to a wide range of health hazards in the workplace, including biological, chemical, physical, ergonomic and psychological hazards. This paper considers the principal occupational exposures of health care workers.

\section{Biologic Hazards}

Human diseases caused by work associated exposure to microbial agents such as bacteria, viruses and fungi. The etiology, pathogenesis, clinical findings, diagnosis and treatment of occupational \& non-occupational infections are the same but there are practical differences in identification of source of exposure, epidemiologic controls and preventive strategies.

Biologic hazards in health care industry include blood borne and air borne pathogens. Most biologic hazards can be classified as infectious or immunologically active. As an example, accidental injection or splash of blood borne viruses (HIV, hepatitis B, hepatitis C) is the major hazard of needle stick injuries especially in laboratory and dialysis staff and medical trainers. HBV is resistant to drying, simple disinfectants, and alcohol and may survive on environmental surfaces for up to one week. Therefore fore, contaminated objects may pose a threat to hospital staffs for several days following last exposure with the infected patient [2]. Preventive strategies include 1) safe collection of fluids and tissues and safe disposal of clothing \& equipment,2) use of Personal protective equipment (Gloves, masks, eye protection, face shield, aprons or gowns), 3)Immunization before and after contact.
Hospital staffs have two to three times the tuberculosis infection rate of non-medical personnel. Laboratory and necropsy room staffs are estimated to be between 100 and 200 times more likely than the general public to develop tuberculosis. Once infection occurs, the organism may disseminate from the lungs to other body sites and the risk for reactivation is highest in the first year after exposure. Hospital staff should be evaluated for Tuberculin Skin test in pre-employment examination. Positive TST shows that the subject had been exposed to TB in the past and is at risk for reactivation, therefore, physical examination and chest radiography should occur to rule out acute clinical infection [3]. Programs to prevent occupational exposure to TB 1)early identification of Infected patients 2)implementing engineering controls such as proper ventilation 3) use of personal protective equipment and 4) medical surveillance.

Health care workers are exposed to many infections disease in workplace including: common cold, influenza, cytomegalovirus, enteric pathogens, herpes simplex virus, measles, mumps, rubella, Varicella, pertussis, scabies, staphylococcus, Center for disease prevention and control recommend hepatitis B, MMR, influenza, Varicella and tetanus vaccination for hospital staffs [4].

\section{Chemical Hazards}

Many chemicals in hospitals are capable of producing adverse health effects through inhalation or by absorption through the skin, which act on the hematopoietic system and damage the lungs, skin, eyes or mucous membranes. Chemical hazards in the workplace for health care workers include exposure to anesthetic gases, antimicrobial drugs, antineoplastic agents, disinfectant agents, ethylene oxide, formaldehyde, glutaraldehyde, latex and solvents [2]. Personnel at operating room, 
delivery area, recovery units have potential exposure to anesthetic gases which have known adverse effects on reproductive system. Regular maintenance and inspections of anesthesia delivery equipment, use of scavenging systems, maintenance of room ventilation rate, and provision of training to staff members could be effective in reduction of exposure [5]. Pharmacists, oncology nurses, housekeepers are at risk of exposure to hazardous drugs, which are prone to health effects on reproductive system and pregnancy outcomes. Implementing vertical flow biological safety cabinets and personal protective equipment are capable in controlling hazardous effects. Disinfectants such as formaldehyde and glutaraldehyde are associated with irritant and allergic effects on skin and respiratory system induced asthma [6].

\section{Physical Hazards}

Potential physical exposures in the hospitals consist of excessive noise in sterilization center, operating room, food service area, engineering unite ,...; temperature extremes in kitchen facilities, boiler room, laundry; ionizing and non-ionizing radiation such as laser and radiofrequency [2]. For example in the use of laser therapies, laser radiation absorption can result in thermal damage when the laser radiation raises the temperature of body tissue, which can result in eye injuries, as well as skin burns and electric shock. Medical evaluation with focus on visual acuity and fundoscopic examination are recommended in pre-employment, after accident and end of employment. Radiation exposure can occur in diagnostic imaging procedure, oncology treatment area and patients treated with radioactive isotope agents [6]. Bodily protection with aprons containing lead is effective.

\section{Ergonomic Hazards}

Ergonomic hazards include heavy lifting, repetitive and forceful movements, and awkward postures that arise from improper work methods and improperly designed workstations and equipment. Some examples of task related risk factor for musculoskeletal injuries are patient transfers, material handling, frequency of lifting, pushing/pulling of objects, and poorly designed work area [5]. These ergonomic hazards can lead to musculoskeletal disorders which can affect the nerves, tendons, muscles and supporting structures of the body. Low back pain and carpal tunnel syndrome are well recognized work related musculoskeletal disorders [2].

\section{Psychosocial hazards}

Violence by both patients and coworkers are one of the psychosocial hazards for health care workers, all violence that hospital staffs experience isn't physical. Verbal violence is a common form of violence, which is due to inadequate staffing, ease of hospital entry, presence of money \& drugs, frustrated family members.

Shift work is one the well-known and inevitable psychosocial hazards in health care setting, which has different health outcomes such as sleep disturbance, psychological disorders, gastrointestinal problems, cardiovascular diseases and aggravated the underlying diseases (epilepsy, diabetes, asthma) [6].

\section{Conclusion}

Health care workers have potential exposure to many occupational hazards which put them at risk of different injuries and disorders. To reduce the risks that threatens the health of this working group, it is important to identify the hazards, and define which hazards are predominant and in priority. Training approaches should be developed and appropriate prevention strategies should be considered to reduce the risks and minimize the hazards [7].

\section{References}

1. WHO (2014) Global strategy on human resources for health: Workforce 2030, WHO Library Cataloguing-inPublication Data 1-64.

2. Rosenstock L, Cullen MR, Brodkin CA, Redlich CA (2005) Textbook of Clinical Occupational and Environmental Medicine. $2^{\text {nd }}$ (Edn.), Elsevier inch.

3. Tuberculosis surveillance protocol for Ontario hospitals (2010) OHA/OMA Communicable Disease Surveillance Protocols, Tuberculosis.

4. Shefer A, Atkinson W, Friedman C, Kuhar DT, Mootrey $\mathrm{G}$, et al. (2011) Immunization of Health-Care Personnel: Recommendations of the Advisory Committee on Immunization Practices (ACIP). Center for disease control and prevention 60(RR07): 1-45.

5. La Dou J (2014) Current Occupational and Environmental Medicine. 5th (Edn.), Mc Grew Hill education. 
6. Walton AL, Rogers B (2017) Workplace Hazards Faced by Nursing Assistants in the United States: A Focused Literature Review. Intl J Environ Res Public Health 14(5): 544.
7. Izadi N, Haghighi KS, Malek M (2015) The Results of Medical Surveillance of Health Care Workers by the First Hospital Occupational Health Clinic. J Mil Med 17(2): 73-79. 